\title{
Organocatalytic Enantioselective Hydrophosphonylation of Aldehydes
}

\author{
Juan V. Alegre-Requena, ${ }^{a}$ Eugenia Marqués-López, ${ }^{a}$ Pablo J. Sanz Miguel ${ }^{b}$ and \\ Raquel P. Herrera $*^{a}$ \\ We report our results concerning the first squaramide-catalysed hydrophosphonylation of \\ aldehydes. In all cases, the reactions proceeded smoothly and cleanly under mild reaction \\ conditions rendering final $\alpha$-hydroxy phosphonates in very good yields and high \\ enantioselectivities. It is one of the scarce organocatalytic examples of this reaction using \\ aldehydes. It is the first time that diphenylphosphite 1e has been successfully employed in a \\ chiral Pudovik reaction with aldehydes, in contrast to the dialkylphosphites used in \\ previously published procedures, extending the generality of this asymmetric methodology.
}

\section{Introduction}

Addition of dialkyl- and diphenylphosphites to carbonyl compounds (Pudovik reaction) ${ }^{1}$ is a powerful and widely applied strategy for the construction of C-P bonds, and a straightforward tool for providing efficient access to chiral $\alpha$ hydroxy phosphonates and their corresponding phosphonic acids. ${ }^{2}$ Interestingly, compounds bearing this motif have shown remarkable biological activities and are currently employed in the pharmaceutical industry (Scheme 1). ${ }^{3}$ The absolute configuration of these phosphonyl compounds could strongly influence their biological activities, ${ }^{4}$ and a growing interest in their asymmetric synthesis has been promoted during the last two decades. Several efficient enantioselective catalytic methods of hydrophosphonylation of aldehydes have been reported in the presence of a chiral metal complex giving access to $\alpha$-hydroxy phosphonates with good outcomes. ${ }^{5,6}$

In addition to the extensive spectrum of biological properties exhibited by this kind of compounds (antibacterial, antivirus, antibiotic and pesticidal activities, as well as a potent antitumor agent), ${ }^{7} \alpha$-hydroxy phosphonates have also attracted attention as synthetic intermediates for preparing further $\alpha_{-}{ }^{8}$ and $\gamma$-functionalised ${ }^{9}$ phosphonates (Scheme 1).

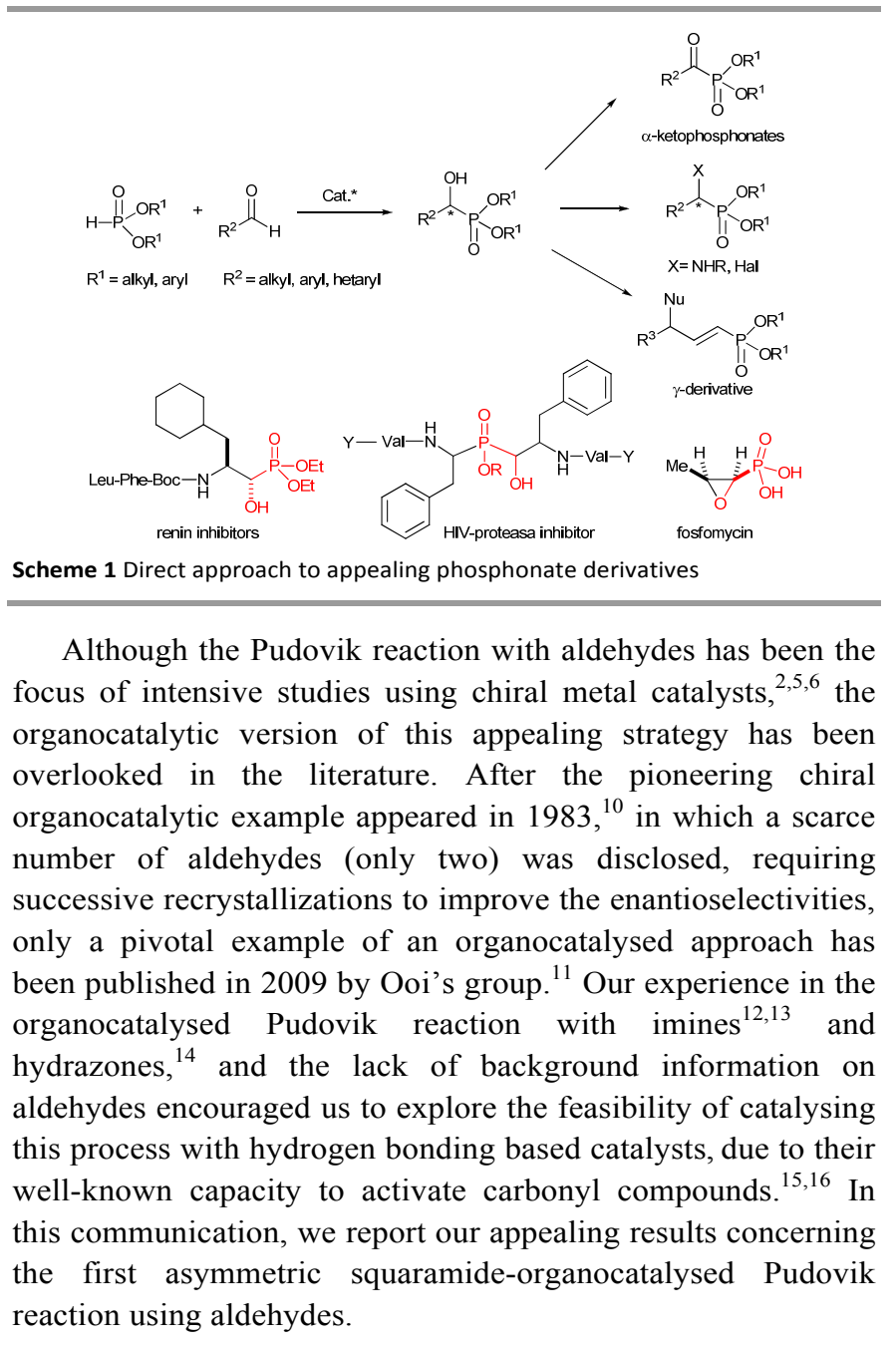




\section{Results and discussion}

We started our investigation examining the viability of the addition of dibenzylphosphite (1a) to aldehyde 2 a catalysed by chiral catalysts 3a-3c acting through hydrogen bonding (Scheme 2). Based on our own experience in the hydrophosphonylation reaction, ${ }^{12,14}$ we foresaw the importance of comprising a basic functionality in the catalyst structure, favouring a plausible bifunctional catalysis, since only model catalysts 3a-c afforded promising results, compared with simple thioureas. $^{17}$

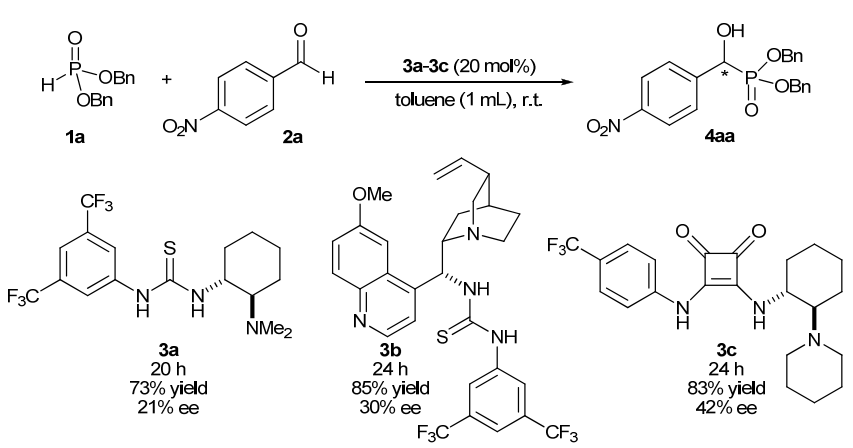

Scheme 2 Model hydrophosphonylation reaction tested

To our delight, the initial screening of catalysts revealed squaramide 3c to be the best compromise in terms of both enantioselectivity and reactivity. However, before discarding thioureas $\mathbf{3 a}$ and $\mathbf{3} \mathbf{b}$, we continued exploring these catalysts to verify if they worked better with other phosphites 1b-1e (Table $1)$.

Table 1. Screening of different phosphites 1b-e with catalysts 3a-c. ${ }^{\text {a }}$

\begin{tabular}{|c|c|c|c|c|c|}
\hline $\begin{array}{c}\mathrm{H}^{\prime} \\
\mathrm{R}=\mathrm{M} \\
i \mathrm{~F}\end{array}$ & ), Et ( $\mathrm{Et}$ & & $\frac{3 a-c \text { (20 }}{\text { toluene }}$ & $N^{-}$ & $\begin{array}{r}0 \\
\text { aa-4ea }\end{array}$ \\
\hline Entry & $\mathrm{R}$ & Cat. & time $(\mathrm{h})$ & yield $(\%)^{b}$ & $\mathrm{ee}(\%)^{c}$ \\
\hline $1^{d}$ & $\mathrm{Me}$ & 3a & 20 & 83 & 8 \\
\hline $2^{d}$ & $\mathrm{Et}$ & $3 a$ & 18 & 90 & 14 \\
\hline $3^{d}$ & $i \operatorname{Pr}$ & $3 a$ & 48 & 83 & 14 \\
\hline $4^{d}$ & $\mathrm{Ph}$ & 3a & 72 & 30 & 18 \\
\hline 5 & $\mathrm{Me}$ & $3 c$ & 20 & 86 & 38 \\
\hline 6 & $\mathrm{Et}$ & $3 c$ & 24 & n.d. ${ }^{e}$ & n.d. ${ }^{e}$ \\
\hline 7 & $i \operatorname{Pr}$ & $3 c$ & 24 & n.d. ${ }^{e}$ & n.d. ${ }^{e}$ \\
\hline 8 & $\mathrm{Ph}$ & $3 c$ & 20 & 83 & 46 \\
\hline 9 & $\mathrm{Ph}$ & $3 b$ & 48 & 57 & 25 \\
\hline
\end{tabular}

${ }^{a}$ Experimental conditions: To a mixture of catalyst 3a-c $(0.02 \mathrm{mmol})$ and aldehyde 2a $(0.1 \mathrm{mmol})$ in toluene $(0.5 \mathrm{~mL})$, phosphite $\mathbf{1 b}-\mathbf{e}(0.2 \mathrm{mmol})$ was further added in a test tube at room temperature. After the reaction time, adducts 4 were isolated by flash chromatography. ${ }^{b}$ Isolated yield. Determined by chiral HPLC analysis. ${ }^{d}$ Reaction performed using $0.2 \mathrm{mmol}$ of aldehyde. ${ }^{e}$ Not determined.

After examining the influence of diverse phosphites 1b-e in our reaction model, and although we did not observe reactivity with some of the phosphites investigated while using catalyst 3c (entries 6 and 7), we found that diphenylphosphite (1e) led to the best results in terms of enantioselectivity and reactivity (entry 8). Subsequent to this exploration, we continued the study with squaramide $3 c$ as the catalyst of choice and phosphite 1e for testing different key parameters such as type of solvent, temperature and concentration (Table 2).

\begin{tabular}{lllllll}
\hline Table 2 Screening of the reaction conditions. ${ }^{a}$ & & & \\
\hline
\end{tabular}

${ }^{a}$ Experimental conditions: To a mixture of catalyst 3c $(0.02 \mathrm{mmol})$ and aldehyde 2a $(0.1 \mathrm{mmol})$ in the corresponding solvent, phosphite 1e $(0.2$ $\mathrm{mmol}$ ) was further added in a test tube at the temperature indicated. After the reaction time, adduct 4 ea was isolated by flash chromatography. ${ }^{b}$ Isolated yield. ${ }^{c}$ Determined by chiral HPLC analysis (Chiralpak IA, flow hexane/AcOEt 70:30, $1 \mathrm{~mL} / \mathrm{min}) .{ }^{d}$ No reaction. ${ }^{e}$ Reaction performed with 1 equiv. of phosphite 1e. ${ }^{f}$ Reaction performed in absence of stirring. 
Among all the studied solvents, we found $\mathrm{CH}_{3} \mathrm{CN}$ to be the best choice for this procedure in terms of enantioselectivity (entry 5), whereas no reaction was observed with some ethereal solvents such as 1,4-dioxane (entry 6) or $\mathrm{Et}_{2} \mathrm{O}$ (entry 7), having a negative influence on the activity of the catalyst. Furthermore, we were pleased to find that cooling the reaction mixture to -38 ${ }^{\circ} \mathrm{C}$ had a remarkable positive effect on the enantioselectivity of the process, without impairing the levels of reactivity (entry 14). We did not decrease the temperature of the reaction any further since we were close to the m.p. of the solvent $\left(-44^{\circ} \mathrm{C}\right)$. The dilution of the reaction also had a positive effect at $-27^{\circ} \mathrm{C}$ on the enantioselectivity of the products (entry 9). Lowering the catalyst loading did not afford better results (entries 13 and 17) and although better yields are attained with a higher catalytic charge as expected (entry 16), we continued with a reasonable 20 mol\%. Surprisingly, we observed that performing the reaction at $-38{ }^{\circ} \mathrm{C}$ in absence of stirring afforded the best results in terms of enantioselectivity and reactivity (compare entries 14 and 15). It seems that the insolubility of the product at this temperature is the driving force of the reaction, since in absence of stirring a big precipitate is observed at the bottom of the reaction tube. This fact resulted in better yields when compared with the same reaction performed at higher temperatures and it also had a clear positive effect on the enantioselectivity. With the optimised reaction conditions in hand, we extended our methodology to a broad range of different substituted aldehydes as shown in Table 3.

Table 3. Scope of the squaramide-catalysed hydrophosphonylation reaction.

\begin{tabular}{|c|c|c|c|c|c|}
\hline \multirow{2}{*}{\multicolumn{2}{|c|}{ 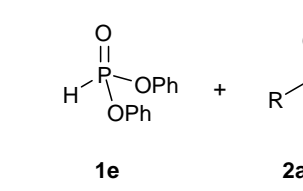 }} & \multicolumn{2}{|c|}{$3 c(20 \mathrm{~mol} \%)$} & \\
\hline & & \multicolumn{2}{|c|}{$\mathrm{CH}_{3} \mathrm{CN},-38^{\circ} \mathrm{C}$} & \multicolumn{2}{|c|}{$\begin{array}{r}\text { II } \\
\text { 4ea-ek }\end{array}$} \\
\hline Entry & $\mathrm{R}$ & Product & $\begin{array}{l}\text { time } \\
\text { (h) }\end{array}$ & $\begin{array}{l}\text { yield } \\
(\%)^{\mathrm{a}}\end{array}$ & $\begin{array}{l}\text { ee } \\
(\%)^{b}\end{array}$ \\
\hline $1^{\mathrm{c}}$ & $4-\mathrm{NO}_{2} \mathrm{Ph}, \mathbf{2 a}$ & 4ea & 95 & 98 & 88 \\
\hline 2 & 4-MePh, $\mathbf{2 b}$ & 4eb & 96 & 81 & 80 \\
\hline 3 & 1-Naphthyl, 2c & $4 e c$ & 92 & 92 & 80 \\
\hline 4 & $\mathrm{Ph}, \mathbf{2 d}$ & 4ed & 92 & 82 & 81 \\
\hline 5 & 4-BrPh, 2e & 4ee & 92 & 72 & 84 \\
\hline 6 & 4-ClPh, 2f & $4 \mathrm{ef}$ & 88 & 80 & 82 \\
\hline 7 & $3-\mathrm{ClPh}, \mathbf{2 g}$ & 4eg & 88 & 98 & 80 \\
\hline $8^{c}$ & 4-CNPh, $\mathbf{2 h}$ & $4 \mathrm{eh}$ & 90 & 98 & 85 \\
\hline 9 & $\mathrm{PhCH}_{2} \mathrm{CH}_{2}, \mathbf{2 i}$ & 4ei & 90 & 77 & 68 \\
\hline 10 & $\mathrm{Cy}, \mathbf{2 j}$ & 4ej & 92 & 98 & 75 \\
\hline 11 & $t \mathrm{Bu}, \mathbf{2 k}$ & 4ek & 94 & 73 & 85 \\
\hline
\end{tabular}

${ }^{a}$ Isolated yield. ${ }^{b}$ Determined by chiral HPLC. ${ }^{c}$ Reaction performed in $1 \mathrm{~mL}$ of solvent.
The addition reaction took place smoothly giving rise to the desired $\alpha$-hydroxy phosphonates $\mathbf{4}$ in excellent yields (up to $98 \%$ ) and high enantioselectivities (up to $88 \%$ ). The efficiency of the developed methodology is well accounted since it was successfully applied to all the aldehydes examined 2a-k. We could reach $88 \%$ and $85 \%$ of ee using aldehydes $2 \mathbf{a}$ and $\mathbf{2 h}$, respectively, simply through slight modifications of the reaction conditions, using $1 \mathrm{~mL}$ of solvent instead of $0.5 \mathrm{~mL}$ (entries 1 and 8). The electronic effects on the enantioselectivity and reactivity were explored using different meta- and parasubstituted benzaldehydes (entries 1-8). Neither the reactivity nor the enantioselectivity suggested a dependence on the electronic environment in the aromatic ring, as excellent results were accomplished with all of them. On the other hand, using aliphatic aldehydes, a clear dependence on steric hindrance could be observed (compare entries 9, 10 and 11), since better enantioselectivities were achieved with bulkier groups, that is from primary $<$ secondary < tertiary carbon, although no correlation with the yield was revealed. In the case of aldehyde $2 \mathbf{k}$, it was necessary to cool the aldehyde before its addition in order to avoid its evaporation. Its volatility could justify the yield reached with this substrate (entry 11).

Since these final adducts have not been previously reported in the chiral version, in order to determine the absolute configuration of our products, single crystals were grown from adducts 4ee and 4eg. The stereochemical outcome was determined to be $R$ for both final products (Figure 1). ${ }^{18}$ The same absolute configuration was assumed for all products 4 .

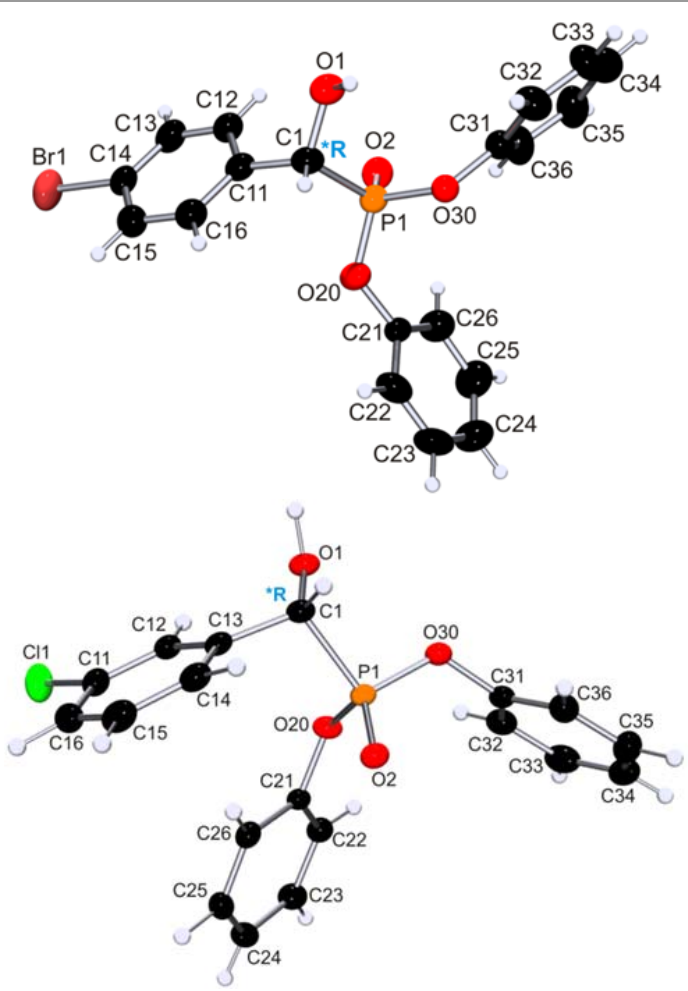

Fig. 1 X-ray crystal structures of $(R)-4$ ee and $(R)-4$ eg. 
Having demonstrated the capacity of squaramide 3c to efficiently catalyse the addition of diphenylphosphite 1e to aldehydes 2a-k, we propose the following reasonable transition state to explain the role of the catalyst based on previous modes of activation by this kind of structure (Figure 2). ${ }^{19}$ On the basis of the experimental results, we envisioned that the chiral squaramide $3 c$ could act in a bifunctional fashion. ${ }^{20}$ On one hand, the aldehyde could be activated by the squaramide moiety through double hydrogen bonding with the NH groups. Simultaneously, the basic nitrogen atom on the pyrrolidine ring would activate the phosphonate form in the tautomeric equilibrium, ${ }^{21}$ driving the attack of the active phosphite form over the $R e$-face of the fixed aldehyde. This would afford the $R$ absolute configuration in all final products, which is consistent with the observed results in our products.

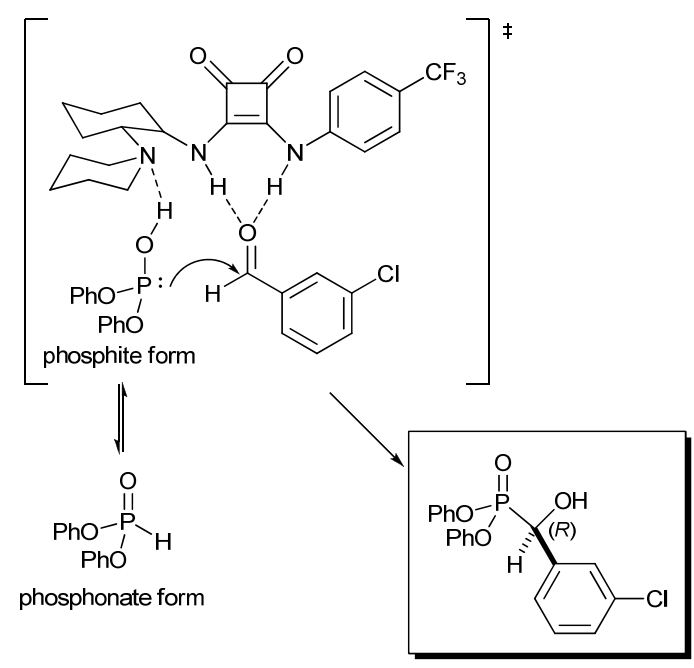

Fig. 2 Proposed mechanistic hypothesis

Our developed methodology, if compared with the pioneering protocol described by Wynberg and co-workers, ${ }^{10,22}$ has broader applicability. It is evident by the fact that our strategy is tolerant towards various functional groups including halides, nitro, cyano, naphthyl and alkyl groups. Additionally, the herein described method is a more straightforward protocol achieving the final goal in a simplest manner, without the necessity of subsequent derivatization or recrystallization in order to obtain high enantioslectivities. Further, our most accessible catalyst 3c compared with that used by Ooi and coworkers and the development of the reaction at $-38^{\circ} \mathrm{C}$ instead of at $-98^{\circ} \mathrm{C}$ makes our procedure an attractive alternative. ${ }^{1 \text { a }}$

\section{Conclusions}

In conclusion, we have demonstrated the ability of the chiral squaramide 3c to accomplish the addition of diphenylphosphite 1e to a variety of aromatic $\mathbf{2 a - h}$ and aliphatic aldehydes $\mathbf{2 i - k}$ through a simple and general approach, with very good results in terms of enantioselectivity and reactivity. This protocol represents an additional extended organocatalytic procedure of hydrophosphonylation of aldehydes, with all reagents and catalyst being commercially available. Moreover, tedious or hazardous conditions, anhydrous solvents or reagents and inert atmosphere are not required as they are in the organometallic version. The potential of this methodology is demonstrated by its simplicity and generality which could become a key work in this area of research. Moreover, it is the first time, to the best of our knowledge, that diphenylphosphite 1e has been successfully employed in a chiral Pudovik reaction of aldehydes in contract to the other dialkylphosphites used in previously published procedures, ${ }^{2}$ extending the generality of this asymmetric methodology. ${ }^{23}$

\section{Experimental Section}

General Experimental Methods. Purification of reaction products was carried out by flash chromatography using silicalgel $(0.063-0.200 \mathrm{~mm})$. Analytical thin layer chromatography was performed on $0.25 \mathrm{~mm}$ silical gel $60-\mathrm{F}$ plates. ESI ionization method and mass analyser type MicroTof-Q were used for the HRMS measurements. ${ }^{1} \mathrm{H}-\mathrm{NMR}$ spectra were recorded at $300 \mathrm{MHz}$ and $400 \mathrm{MHz} ;{ }^{13} \mathrm{C}-\mathrm{NMR}$ spectra were recorded at $75 \mathrm{MHz}$ and $100 \mathrm{MHz} ; \mathrm{CDCl}_{3}$ as the solvent. Chemical shifts were reported in the $\delta$ scale relative to residual $\mathrm{CHCl}_{3}(7.26 \mathrm{ppm})$ for ${ }^{1} \mathrm{H}-\mathrm{NMR}$ and to the central line of $\mathrm{CDCl}_{3}$ (77 ppm) for ${ }^{13} \mathrm{C}-\mathrm{NMR}$.

Materials. All commercially available solvents and reagents were used as received. The ${ }^{1} \mathrm{H}$ and ${ }^{13} \mathrm{C}$ NMR spectra for compounds $\mathbf{4 e b},{ }^{24} \mathbf{4 e c},{ }^{25} \mathbf{4 e e},{ }^{25} \mathbf{4 e g},{ }^{25}$ are consistent with values previously reported in the literature

\section{Representative procedure for squaramide-organocatalysed} hydrophosphonylation reaction of aldehydes

To a mixture of catalyst $3 \mathbf{c}(0.02 \mathrm{mmol})$, and aldehyde $\mathbf{2 a - k}$ $(0.1 \mathrm{mmol})$ in $\mathrm{CH}_{3} \mathrm{CN}(0.5 \mathrm{~mL})$, phosphite $1 \mathrm{e}(0.2 \mathrm{mmol})$ was further added in a test tube at $-38{ }^{\circ} \mathrm{C}$. After the reaction time (see Table 3 ), adducts 4 were isolated by flash chromatography or medium pressure liquid chromatography $\left(\mathrm{SiO}_{2}\right.$, hexane/EtOAc 7:3). Yields and enantioselectivities are reported in Table 3.

\section{(R)-Diphenyl hydroxy(4-nitrophenyl)methylphosphonate (4ea)}

Following the general procedure, compound 4ea was obtained after $95 \mathrm{~h}$ of reaction at $-38{ }^{\circ} \mathrm{C}$ as a white solid in $98 \%$ yield $(37.7 \mathrm{mg})$. The ee of the product was determined to be $88 \%$ by HPLC using a Daicel Chiralpak IA column $(n$-hexane/AcOEt $=$ 70:30, flow rate $1 \mathrm{~mL} / \mathrm{min}, \lambda=268.3 \mathrm{~nm}): \tau_{\text {major }}=23.7 \mathrm{~min}$; $\tau_{\text {minor }}=20.7$ min. M.p. $137-140{ }^{\circ} \mathrm{C} .[\alpha]_{\mathrm{D}}{ }^{26}=+47\left(c 0.40, \mathrm{CHCl}_{3}\right.$, $80 \%$ ee). ${ }^{1} \mathrm{H}$ NMR $\left(400 \mathrm{MHz}, \mathrm{CDCl}_{3}\right) \delta 8.24(\mathrm{~d}, J=8.1 \mathrm{~Hz}$, $2 \mathrm{H}), 7.76(\mathrm{dd}, J=2.4,8.4 \mathrm{~Hz}, 2 \mathrm{H}), 7.27-7.31(\mathrm{~m}, 4 \mathrm{H}), 7.16-$ $7.20(\mathrm{~m}, 2 \mathrm{H}), 7.03-7.08(\mathrm{~m}, 4 \mathrm{H}), 5.44(\mathrm{dd}, J=4.8,11.0 \mathrm{~Hz}$, $1 \mathrm{H}), 4.02(\mathrm{dd}, J=5.3,7.8 \mathrm{~Hz}, 1 \mathrm{H}) .{ }^{13} \mathrm{C} \mathrm{NMR}\left(75 \mathrm{MHz}, \mathrm{CDCl}_{3}\right)$ $\delta 150.0(\mathrm{~d}, J=10.1 \mathrm{~Hz}, 1 \mathrm{C}), 149.9(\mathrm{~d}, J=10.6 \mathrm{~Hz}, 1 \mathrm{C}), 147.7$ (d, $J=4.0 \mathrm{~Hz}, 1 \mathrm{C}), 143.0$ (d, $J=1.8 \mathrm{~Hz}, 1 \mathrm{C}), 129.9$ (d, $J=6.6$ $\mathrm{Hz}, 4 \mathrm{C}), 128.0$ (d, $J=1.8 \mathrm{~Hz}, 2 \mathrm{C}), 125.7$ (d, $J=7.6 \mathrm{~Hz}, 2 \mathrm{C}$ ), 
123.4 (d, $J=2.8 \mathrm{~Hz}, 2 \mathrm{C}), 120.5$ (d, $J=9.3 \mathrm{~Hz}, 2 \mathrm{C}), 120.4$ (d, $J$ $=9.3 \mathrm{~Hz}, 2 \mathrm{C}), 69.8(\mathrm{~d}, J=160.3 \mathrm{~Hz}, 1 \mathrm{C}) . \mathrm{IR}(\mathrm{KBr}$ film $)\left(\mathrm{cm}^{-1}\right)$ $v$ 3306, 2923, 2853, 1588, 1513, 1488, 1377, 1215, 1182, 945, 761, 690. HRMS (ESI+) calcd $\mathrm{C}_{19} \mathrm{H}_{16} \mathrm{NNaO}_{6} \mathrm{P} 408.0613$; found $408.0607[\mathrm{M}+\mathrm{Na}]$.

\section{(R)-Diphenyl hydroxy(p-tolyl)methylphosphonate (4eb) $)^{24}$}

Following the general procedure, compound 4eb was obtained after $96 \mathrm{~h}$ of reaction at $-38{ }^{\circ} \mathrm{C}$ as a white solid in $81 \%$ yield $(28.7 \mathrm{mg})$. The ee of the product was determined to be $80 \%$ by HPLC using a Daicel Chiralpak IC column ( $n$-hexane $/ i-\mathrm{PrOH}=$ 90:10, flow rate $1 \mathrm{~mL} / \mathrm{min}, \lambda=262 \mathrm{~nm}): \tau_{\text {major }}=18.9 \mathrm{~min} ; \tau_{\text {minor }}$ $=16.8 \mathrm{~min} .[\alpha]_{\mathrm{D}}{ }^{28}=+27\left(c 0.87, \mathrm{CHCl}_{3}, 74 \%\right.$ ee $)$. IR $(\mathrm{KBr}$ film) $\left(\mathrm{cm}^{-1}\right) \vee 3337,2924,2854,1590,1489,1456,1377,1260$, $1241,1212,1190,1163,1071,1022,1040,940,800,764,689$. HRMS (ESI+) calcd $\mathrm{C}_{20} \mathrm{H}_{19} \mathrm{NaO}_{4} \mathrm{P}$ 377.0919; found 377.0913 $[\mathrm{M}+\mathrm{Na}]$.

\section{(R)-Diphenyl hydroxy(naphthalen-1-yl)methylphosphonate} (4ec)

Following the general procedure, compound 4ec was obtained after $92 \mathrm{~h}$ of reaction at $-38{ }^{\circ} \mathrm{C}$ as a white solid in $92 \%$ yield ( $35.9 \mathrm{mg}$ ). The ee of the product was determined to be $80 \%$ by HPLC using a Daicel Chiralpak IC column $(n$-hexane $/ i-\mathrm{PrOH}=$ 90:10, flow rate $1 \mathrm{~mL} / \mathrm{min}, \lambda=283.7 \mathrm{~nm}): \tau_{\text {major }}=21.5 \mathrm{~min}$; $\tau_{\text {minor }}=17.3$ min. M.p. $110-112^{\circ} \mathrm{C} .[\alpha]_{\mathrm{D}}{ }^{26}=+98\left(c 0.61, \mathrm{CHCl}_{3}\right.$, $80 \%$ ee). ${ }^{1} \mathrm{H}$ NMR (400 MHz, $\left.\mathrm{CDCl}_{3}\right) \delta 8.16(\mathrm{~d}, J=8.5 \mathrm{~Hz}$, $1 \mathrm{H}), 7.97(\mathrm{dd}, J=3.4,7.2 \mathrm{~Hz}, 1 \mathrm{H}), 7.85-7.90(\mathrm{~m}, 2 \mathrm{H}), 7.49$ $7.56(\mathrm{~m}, 3 \mathrm{H}), 7.04-7.27(\mathrm{~m}, 8 \mathrm{H}), 6.88-6.90(\mathrm{~m}, 2 \mathrm{H}), 6.20$ (dd, $J$ $=3.8,10.5 \mathrm{~Hz}, 1 \mathrm{H}), 3.56(\mathrm{br} \mathrm{s}, 1 \mathrm{H}) .{ }^{13} \mathrm{C} \mathrm{NMR}(100 \mathrm{MHz}$, $\left.\mathrm{CDCl}_{3}\right) \delta 150.3(\mathrm{~d}, J=10.3 \mathrm{~Hz}, 1 \mathrm{C}), 150.3(\mathrm{~d}, J=9.9 \mathrm{~Hz}, 1 \mathrm{C})$, 133.7 (d, $J=1.7 \mathrm{~Hz}, 1 \mathrm{C}), 131.5$ (d, $J=1.8 \mathrm{~Hz}, 1 \mathrm{C}), 130.9$ (d, $J$ $=6.8 \mathrm{~Hz}, 1 \mathrm{C}), 129.6(\mathrm{~d}, J=14.5 \mathrm{~Hz}, 4 \mathrm{C}), 129.4(\mathrm{~d}, J=3.5 \mathrm{~Hz}$, 1C), 128.8 (d, $J=0.7 \mathrm{~Hz}, 1 \mathrm{C}), 126.5$ (1C), 126.1 (d, $J=6.4 \mathrm{~Hz}$, 1C), 125.8 (1C), 125.4 (d, $J=3.5 \mathrm{~Hz}, 1 \mathrm{C}), 125.2$ (d, $J=14.9$ $\mathrm{Hz}, 2 \mathrm{C}$ ), 123.4 (1C), 120.4 (d, $J=22.4 \mathrm{~Hz}, 2 \mathrm{C}), 120.3$ (d, $J=$ $22.4 \mathrm{~Hz}, 2 \mathrm{C}), 67.2(\mathrm{~d}, J=163.7 \mathrm{~Hz}, 1 \mathrm{C})$. IR ( $\mathrm{KBr}$ film) $\left(\mathrm{cm}^{-1}\right)$ v 3252, 2924, 2854, 1590, 1513, 1488, 1463, 1377, 1250, 1186, 1161, 942, 804, 767, 688. HRMS (ESI+) calcd $\mathrm{C}_{23} \mathrm{H}_{19} \mathrm{NaO}_{4} \mathrm{P}$ 413.0919; found 413.0913 [M+Na].

\section{(R)-Diphenyl hydroxy(phenyl)methylphosphonate (4ed) ${ }^{25}$}

Following the general procedure, compound 4ed was obtained after $92 \mathrm{~h}$ of reaction at $-38{ }^{\circ} \mathrm{C}$ in $82 \%$ yield $(27.9 \mathrm{mg})$. The ee of the product was determined to be $81 \%$ by HPLC using a Daicel Chiralpak IC column ( $n$-hexane $/ i-\mathrm{PrOH}=90: 10$, flow rate $1 \mathrm{~mL} / \mathrm{min}, \lambda=261.2 \mathrm{~nm}): \tau_{\text {major }}=16.9 \mathrm{~min} ; \tau_{\text {minor }}=14.4$ $\min .[\alpha]_{\mathrm{D}}^{28}=+21\left(c 0.35, \mathrm{CHCl}_{3}, 79 \%\right.$ ee $)$.

\section{(R)-Diphenyl (4-bromophenyl)(hydroxy)methylphosphonate (4ee)}

Following the general procedure, compound 4ee was obtained after $92 \mathrm{~h}$ of reaction at $-38{ }^{\circ} \mathrm{C}$ as a white solid in $72 \%$ yield $(30.2 \mathrm{mg})$. The ee of the product was determined to be $84 \%$ by HPLC using a Daicel Chiralpak IA column ( $n$-hexane/ $i$-PrOH $=90: 10$, flow rate $1 \mathrm{~mL} / \mathrm{min}, \lambda=260.7 \mathrm{~nm}): \tau_{\text {major }}=21.1 \mathrm{~min}$; $\tau_{\text {minor }}=19.3$ min. M.p. $128-130^{\circ} \mathrm{C} .[\alpha]_{\mathrm{D}}{ }^{23}=+29\left(c 0.47, \mathrm{CHCl}_{3}\right.$, $83 \%$ ee). ${ }^{1} \mathrm{H}$ NMR (400 MHz, $\left.\mathrm{CDCl}_{3}\right) \delta 7.49-7.51(\mathrm{~m}, 2 \mathrm{H})$, 7.42-7.46 (m, 2H), 7.25-7.30 (m, 4H), 7.13-7.19 (m, 2H), 7.06$7.08(\mathrm{~m}, 2 \mathrm{H}), 7.01-7.03(\mathrm{~m}, 2 \mathrm{H}), 5.25(\mathrm{~d}, J=9.3 \mathrm{~Hz}, 1 \mathrm{H}) .{ }^{13} \mathrm{C}$ NMR $\left(100 \mathrm{MHz}, \mathrm{CDCl}_{3}\right) \delta 150.2(\mathrm{~d}, J=10.4 \mathrm{~Hz}, 1 \mathrm{C}), 150.1$ (d, $J=11.1 \mathrm{~Hz}, 1 \mathrm{C}), 134.8$ (1C), $131.5(\mathrm{~d}, J=2.6 \mathrm{~Hz}, 2 \mathrm{C})$, 129.7 (d, $J=8.3 \mathrm{~Hz}, 4 \mathrm{C}), 129.0$ (d, $J=6.1 \mathrm{~Hz}, 2 \mathrm{C}), 125.3$ (d, $J$ $=10.4 \mathrm{~Hz}, 2 \mathrm{C}), 122.5(\mathrm{~d}, J=4.3 \mathrm{~Hz}, 2 \mathrm{C}), 120.5(\mathrm{~d}, J=4.6 \mathrm{~Hz}$, 2C), 120.5 (d, $J=4.8 \mathrm{~Hz}, 2 \mathrm{C}), 69.9$ (d, $J=161.9 \mathrm{~Hz}, 1 \mathrm{C})$. IR $(\mathrm{KBr}$ film $)\left(\mathrm{cm}^{-1}\right) \vee 3319,2924,2854,1730,1589,1511,1487$, $1462,1403,1377,1242,1212,1189,1163,1101,1070,1043$, $1025,1010,942,823,766,730,689,616,581,442$. HRMS (ESI+) calcd $\mathrm{C}_{19} \mathrm{H}_{16} \mathrm{BrNaO}_{4} \mathrm{P} \quad 440.9867$; found 440.9862 $[\mathrm{M}+\mathrm{Na}]$.

\section{(R)-Diphenyl (4-chlorophenyl)(hydroxy)methylphosphonate (4ef) $^{25}$}

Following the general procedure, compound 4ef was obtained after $88 \mathrm{~h}$ of reaction at $-38{ }^{\circ} \mathrm{C}$ as a white solid in $80 \%$ yield $(30 \mathrm{mg})$. The ee of the product was determined to be $82 \%$ by HPLC using a Daicel Chiralpak IC column ( $n$-hexane $/ i-\mathrm{PrOH}=$ 98:2, flow rate $1 \mathrm{~mL} / \mathrm{min}, \lambda=261.3 \mathrm{~nm}): \tau_{\text {major }}=12.2 \mathrm{~min}$; $\tau_{\text {minor }}=10.3 \mathrm{~min} .[\alpha]_{\mathrm{D}}{ }^{28}=+18\left(c 0.32, \mathrm{CHCl}_{3}, 78 \%\right.$ ee $) . \mathrm{IR}$ $(\mathrm{KBr}$ film $)\left(\mathrm{cm}^{-1}\right)$ v 3313, 2924, 2853, 1589, 1489, 1457, 1377, $1185,1162,1014,759,687$.

\section{(R)-Diphenyl (3-chlorophenyl)(hydroxy)methylphosphonate (4eg)}

Following the general procedure, compound 4eg was obtained after $88 \mathrm{~h}$ of reaction at $-38{ }^{\circ} \mathrm{C}$ as a white solid in $98 \%$ yield $(36.7 \mathrm{mg})$. The ee of the product was determined to be $80 \%$ by HPLC using a Daicel Chiralpak IC column ( $n$-hexane $/ i-\mathrm{PrOH}=$ 95:5, flow rate $1 \mathrm{~mL} / \mathrm{min}, \lambda=272.6 \mathrm{~nm}): \tau_{\text {major }}=21.7 \mathrm{~min}$; $\tau_{\text {minor }}=16.5$ min. M.p. $154-156{ }^{\circ} \mathrm{C} . \quad[\alpha]_{\mathrm{D}}{ }^{28}=+26(c 0.33$, $\mathrm{CHCl}_{3}, 77 \%$ ee). ${ }^{1} \mathrm{H}$ NMR $\left(400 \mathrm{MHz}, \mathrm{CDCl}_{3}\right) \delta 7.60-7.61(\mathrm{~m}$, $1 \mathrm{H})$, 7.46-7.51 (m, 1H), 7.26-7.35 (m, 6H), 7.15-7.21 (m, 2H), 7.04-7.11 (m, 4H), $5.35(\mathrm{dd}, J=5.0,9.6 \mathrm{~Hz}, 1 \mathrm{H}), 3.46(\mathrm{dd}, J=$ $5.1,10.0 \mathrm{~Hz}, 1 \mathrm{H}) .{ }^{13} \mathrm{C}$ NMR $\left(75 \mathrm{MHz}, \mathrm{CDCl}_{3}\right) \delta 150.2(\mathrm{~d}, J=$ $10.0 \mathrm{~Hz}, 1 \mathrm{C}), 150.1(\mathrm{~d}, J=10.2 \mathrm{~Hz}, 1 \mathrm{C}), 137.4(\mathrm{~d}, J=2.2 \mathrm{~Hz}$, 1C), 134.4 (d, $J=3.0 \mathrm{~Hz}, 1 \mathrm{C}), 129.7$ (d, $J=4.4 \mathrm{~Hz}, 4 \mathrm{C}), 129.7$ (1C), 128.7 (d, $J=3.4 \mathrm{~Hz}, 1 \mathrm{C}), 127.5$ (d, $J=6.3 \mathrm{~Hz}, 1 \mathrm{C}), 125.5$ (d, $J=5.8 \mathrm{~Hz}, 1 \mathrm{C}), 125.4$ (d, $J=5.3 \mathrm{~Hz}, 2 \mathrm{C}), 120.5$ (d, $J=6.6$ $\mathrm{Hz}, 2 \mathrm{C}), 120.5$ (d, $J=6.6 \mathrm{~Hz}, 2 \mathrm{C}), 70.1$ (d, $J=160.8 \mathrm{~Hz}, 1 \mathrm{C})$. IR $(\mathrm{KBr}$ film $)\left(\mathrm{cm}^{-1}\right)$ v 3343, 2924, 2853, 1723, 1589, 1490, 1456, 1377, 1252, 1240, 1208, 1196, 1184, 1166, 1099, 1071, 1010, 960, 938, 808, 769, 759, 690. HRMS (ESI+) calcd $\mathrm{C}_{19} \mathrm{H}_{16} \mathrm{ClNaO}_{4} \mathrm{P}$ 397.0372; found 397.0367 [M+Na].

\section{(R)-Diphenyl (4-cyanophenyl)(hydroxy)methylphosphonate} (4eh)

Following the general procedure, compound 4eh was obtained after $90 \mathrm{~h}$ of reaction at $-38{ }^{\circ} \mathrm{C}$ as a white solid in $98 \%$ yield $(35.8 \mathrm{mg})$. The ee of the product was determined to be $85 \%$ by HPLC using a Daicel Chiralpak IA column $(n$-hexane $/ i-\mathrm{PrOH}=$ 90:10, flow rate $1 \mathrm{~mL} / \mathrm{min}, \lambda=236.4 \mathrm{~nm}): \tau_{\text {major }}=31.7 \mathrm{~min}$; $\tau_{\text {minor }}=27.8$ min. M.p. $124-126^{\circ} \mathrm{C} .[\alpha]_{\mathrm{D}}{ }^{30}=+51\left(c 1.31, \mathrm{CHCl}_{3}\right.$, 
$82 \%$ ee). ${ }^{1} \mathrm{H}$ NMR (400 MHz, $\left.\mathrm{CDCl}_{3}\right) \delta$ 7.64-7.70 (m, 4H), 7.26-7.31 (m, 4H), 7.15-7.20 (m, 2H), 7.01-7.06 (m, 4H), 5.34 $(\mathrm{dd}, J=5.1,10.7 \mathrm{~Hz}, 1 \mathrm{H}), 4.52(\mathrm{t}, J=6.4 \mathrm{~Hz}, 1 \mathrm{H}) .{ }^{13} \mathrm{C} \mathrm{NMR}$ $\left(100 \mathrm{MHz}, \mathrm{CDCl}_{3}\right) \delta 150.0(\mathrm{~d}, J=13.4 \mathrm{~Hz}, 1 \mathrm{C}), 149.9(\mathrm{~d}, J=$ $13.9 \mathrm{~Hz}, 1 \mathrm{C}), 140.8(\mathrm{~d}, J=2.4 \mathrm{~Hz}, 1 \mathrm{C}), 132.1(\mathrm{~d}, J=2.9 \mathrm{~Hz}$, 2C), 129.8 (d, $J=7.2 \mathrm{~Hz}, 4 \mathrm{C}$ ), 127.9 (d, $J=5.7 \mathrm{~Hz}, 2 \mathrm{C}$ ), 125.6 (d, $J=9.8 \mathrm{~Hz}, 2 \mathrm{C}), 120.4$ (d, $J=4.2 \mathrm{~Hz}, 2 \mathrm{C}), 120.4$ (d, $J=4.1$ $\mathrm{Hz}, 2 \mathrm{C}), 118.5$ (d, $J=2.2 \mathrm{~Hz}, 1 \mathrm{C}), 112.2$ (d, $J=3.8 \mathrm{~Hz}, 1 \mathrm{C})$, $70.0(\mathrm{~d}, J=159.8 \mathrm{~Hz}, 1 \mathrm{C})$. IR ( $\mathrm{KBr}$ film) $\left(\mathrm{cm}^{-1}\right) v 3319,2924$, 2854, 2225, 1641, 1529, 1491, 1462, 1377, 1245, 1208, 1184, 1069, 940, 757, 685. HRMS (ESI+) calcd $\mathrm{C}_{20} \mathrm{H}_{16} \mathrm{NNaO}_{4} \mathrm{P}$ 388.0715; found 388.0709 [M+Na].

\section{(R)-Diphenyl 1-hydroxy-3-phenylpropylphosphonate (4ei)}

Following the general procedure, compound 4ei was obtained as a white solid after $90 \mathrm{~h}$ of reaction at $-38{ }^{\circ} \mathrm{C}$ as a white solid in $77 \%$ yield $(28.4 \mathrm{mg})$. The ee of the product was determined to be $68 \%$ by HPLC using a Daicel Chiralpak IA column ( $n$ hexane $/ i-\mathrm{PrOH}=90: 10$, flow rate $1 \mathrm{~mL} / \mathrm{min}, \lambda=261.2 \mathrm{~nm}$ ): $\tau_{\text {major }}=13.1 \mathrm{~min} ; \tau_{\text {minor }}=14.7$ min. M.p. $108-110^{\circ} \mathrm{C} .[\alpha]_{\mathrm{D}}{ }^{25}=-$ 6.8 (c 1.02, $\mathrm{CHCl}_{3}, 67 \%$ ee). ${ }^{1} \mathrm{H}$ NMR (400 $\left.\mathrm{MHz}, \mathrm{CDCl}_{3}\right) \delta$ 7.12-7.32 (m, 15H), 4.15-4.19 (m, 1H), 3.16 (br s, 1H), 2.98$3.04(\mathrm{~m}, 1 \mathrm{H}), 2.77-2.85(\mathrm{~m}, 1 \mathrm{H}), 2.17-2.32(\mathrm{~m}, 2 \mathrm{H}) .{ }^{13} \mathrm{C} \mathrm{NMR}$ $\left(100 \mathrm{MHz}, \mathrm{CDCl}_{3}\right) \delta 150.3(\mathrm{~d}, J=9.1 \mathrm{~Hz}, 1 \mathrm{C}), 150.2(\mathrm{~d}, J=$ $10.0 \mathrm{~Hz}, 1 \mathrm{C}), 140.9$ (1C), 129.8 (4C), 128.6 (d, $J=13.0 \mathrm{~Hz}$, 4C), $126.2(1 \mathrm{C}), 125.3(\mathrm{~d}, J=5.0 \mathrm{~Hz}, 1 \mathrm{C}), 125.3(\mathrm{~d}, J=4.9 \mathrm{~Hz}$, 1C), 120.7 (d, $J=5.9 \mathrm{~Hz}, 2 \mathrm{C}), 120.7$ (d, $J=5.9 \mathrm{~Hz}, 2 \mathrm{C}), 66.8$ $(\mathrm{d}, J=160.6 \mathrm{~Hz}, 1 \mathrm{C}), 33.0(\mathrm{~d}, J=2.0 \mathrm{~Hz}, 1 \mathrm{C}), 31.6(\mathrm{~d}, J=15.0$ $\mathrm{Hz}, 1 \mathrm{C})$. IR (KBr film) $\left(\mathrm{cm}^{-1}\right) \vee 3338,3062,3026,2926,2856$, 2225, 1591, 1490, 1454, 1188, 1162, 1070, 1025, 1008, 941, 762, 689. HRMS (ESI+) calcd $\mathrm{C}_{21} \mathrm{H}_{21} \mathrm{NaO}_{4} \mathrm{P}$ 391.1075; found $391.1070[\mathrm{M}+\mathrm{Na}]$. $^{\prime}$

\section{(R)-Diphenyl cyclohexyl(hydroxy)methylphosphonate (4ej)}

Following the general procedure, compound 4ej was obtained after $92 \mathrm{~h}$ of reaction at $-38{ }^{\circ} \mathrm{C}$ as a white solid in $98 \%$ yield $(33.9 \mathrm{mg}$ ). The ee of the product was determined to be $75 \%$ by HPLC using a Daicel Chiralpak IA column $(n$-hexane $/ i-\mathrm{PrOH}=$ 95:5, flow rate $1 \mathrm{~mL} / \mathrm{min}, \lambda=262.4 \mathrm{~nm}): \tau_{\text {major }}=22.1 \mathrm{~min}$; $\tau_{\text {minor }}=26.2 \mathrm{~min}$. M.p. $100-102{ }^{\circ} \mathrm{C} .[\alpha]_{\mathrm{D}}{ }^{26}=-3\left(c 0.96, \mathrm{CHCl}_{3}\right.$, $69 \%$ ee). ${ }^{1} \mathrm{H}$ NMR (400 MHz, $\left.\mathrm{CDCl}_{3}\right) \delta$ 7.29-7.34 (m, 4H), $7.15-7.21(\mathrm{~m}, 6 \mathrm{H}), 4.02(\mathrm{q}, J=5.7,12.8 \mathrm{~Hz}, 1 \mathrm{H}), 2.47(\mathrm{dd}, J=$ $6.5,7.2 \mathrm{~Hz}, 1 \mathrm{H}), 2.10-2.13(\mathrm{~m}, 1 \mathrm{H}), 1.95-2.06(\mathrm{~m}, 1 \mathrm{H}), 1.88-$ $1.91(\mathrm{~m}, 1 \mathrm{H}), 1.79-1.81(\mathrm{~m}, 2 \mathrm{H}), 1.66-1.70(\mathrm{~m}, 1 \mathrm{H}), 1.13-1.46$ $(\mathrm{m}, 5 \mathrm{H}) .{ }^{13} \mathrm{C}$ NMR $\left(100 \mathrm{MHz}, \mathrm{CDCl}_{3}\right) \delta 150.3(\mathrm{~d}, J=10.1 \mathrm{~Hz}$, 1C), 150.2 (d, $J=9.8 \mathrm{~Hz}, 1 \mathrm{C}), 129.7$ (d, $J=5.7 \mathrm{~Hz}, 4 \mathrm{C}), 125.2$ (m, 2C), 120.6 (d, $J=4.0 \mathrm{~Hz}, 2 \mathrm{C}), 120.6(\mathrm{~d}, J=4.0 \mathrm{~Hz}, 2 \mathrm{C})$, 72.6 (d, $J=155.1 \mathrm{~Hz}, 1 \mathrm{C}), 39.8$ (d, $J=2.4 \mathrm{~Hz}, 1 \mathrm{C}), 29.9$ (d, $J$ $=9.5 \mathrm{~Hz}, 1 \mathrm{C}), 27.9(\mathrm{~d}, J=7.6 \mathrm{~Hz}, 1 \mathrm{C}), 26.1$ (1C), 25.9 (d, $J=$ $17.6 \mathrm{~Hz}, 2 \mathrm{C})$. IR (KBr film) $\left(\mathrm{cm}^{-1}\right) \vee 3325,2932,2852,1591$, 1487, 1448, 1408, 1193, 1161, 1106, 1070, 1003, 972, 935, 802, 774, 690, 661, 475. HRMS (ESI+) calcd $\mathrm{C}_{19} \mathrm{H}_{23} \mathrm{NaO}_{4} \mathrm{P}$ 369.1232; found $369.1226[\mathrm{M}+\mathrm{Na}]$.

\section{(R)-Diphenyl-1-hydroxy-2,2-dimethylpropylphosphonate (4ek)}

Following the general procedure, compound 4ek was obtained after $94 \mathrm{~h}$ of reaction at $-38{ }^{\circ} \mathrm{C}$ as a white solid in $73 \%$ yield (23.4 $\mathrm{mg}$ ). The ee of the product was determined to be $85 \%$ by HPLC using a Daicel Chiralpak IA column ( $n$-hexane/ $i$-PrOH $=98: 2$, flow rate $1 \mathrm{~mL} / \mathrm{min}, \lambda=262.4 \mathrm{~nm}): \tau_{\text {major }}=28.2 \mathrm{~min}$; $\tau_{\text {minor }}=32.6$ min. M.p. $116-120^{\circ} \mathrm{C} .[\alpha]_{\mathrm{D}}{ }^{25}=+7(c 0.47$, acetone, $84 \%$ ee). ${ }^{1} \mathrm{H}$ NMR (400 MHz, $\left.\mathrm{CDCl}_{3}\right) \delta$ 7.28-7.34 (m, 4H), 7.14-7.20 (m, 6H), $3.92(\mathrm{dd}, J=6.4,7.2 \mathrm{~Hz}, 1 \mathrm{H}), 2.59$ (dd, $J=$ 6.8, 7.8 Hz, 1H), $1.22(\mathrm{~s}, 9 \mathrm{H}) .{ }^{13} \mathrm{C}$ NMR $\left(100 \mathrm{MHz}, \mathrm{CDCl}_{3}\right) \delta$ 150.3 (d, $J=22.2 \mathrm{~Hz}, 1 \mathrm{C}), 150.2$ (d, $J=21.9 \mathrm{~Hz}, 1 \mathrm{C}), 129.7$ (d, $J=7.6 \mathrm{~Hz}, 4 \mathrm{C}), 125.2(\mathrm{~d}, J=3.5 \mathrm{~Hz}, 1 \mathrm{C}), 125.1(\mathrm{~d}, J=3.5 \mathrm{~Hz}$, 1C), $120.6(\mathrm{~d}, J=4.0 \mathrm{~Hz}, 2 \mathrm{C}), 120.6(\mathrm{~d}, J=4.1 \mathrm{~Hz}, 2 \mathrm{C}), 76.3$ (d, $J=152.5 \mathrm{~Hz}, 1 \mathrm{C}), 35.0$ (d, $J=3.6 \mathrm{~Hz}, 1 \mathrm{C}), 26.7$ (d, $J=6.7$ $\mathrm{Hz}, 3 \mathrm{C})$. IR (KBr film) $\left(\mathrm{cm}^{-1}\right)$ v 3357, 2924, 2854, 1590, 1490 , 1462, 1377, 1246, 1205, 1189, 1161, 1064, 945, 903, 802, 766, 690. HRMS (ESI+) calcd $\mathrm{C}_{17} \mathrm{H}_{21} \mathrm{NaO}_{4} \mathrm{P}$ 343.1075; found $343.1070\left[\mathrm{M}^{+}+\mathrm{Na}\right]$.

\section{Acknowledgements}

We thank the Spanish Ministry of Economía y Competitividad (MEC CTQ2010-19606 and CTQ2011-27593) and the Government of Aragón (Zaragoza. Spain. Research Group E10) for financial support of our research. P. J. S. M. acknowledges funding through the "Ramón y Cajal" program (MEC).

\section{Notes and references}

${ }^{a}$ Laboratorio de Síntesis Asimétrica, Departamento de Química Orgánica. Instituto de Síntesis Química y Catálisis Homogénea (ISQCH). CSICUniversidad de Zaragoza. E-50009 Zaragoza, Spain Fax: +34 976762075. Phone: +34 976762281. E-mail: raquelph@unizar.es

${ }^{b}$ Departamento de Química Inorgánica. Instituto de Síntesis Química y Catálisis Homogénea (ISQCH), CSIC-Universidad de Zaragoza. E-50009 Zaragoza, Spain.

Electronic Supplementary Information (ESI) available: General experimental information, ${ }^{1} \mathrm{H}$ and ${ }^{13} \mathrm{C}$ NMR spectra and HPLC chromatograms for all final products 4ea-ek. See DOI: $10.1039 / \mathrm{b} 000000 \mathrm{x} /$

1 A. N. Pudovik and I. V. Konovalova, Synthesis, 1979, 81.

2 a) H. Gröger and B. Hammer, Chem. Eur. J., 2000, 6, 943; b) D. Enders, A. Saint-Dizier, M.-I. Lannou and A. Lenzen, Eur. J. Org. Chem., 2006, 29; c) P. Merino, E. Marqués-López and R. P. Herrera, Adv. Synth. Catal., 2008, 350, 1195; d) L. Albrecht, A. Albrecht, H. Krawczyk and K. A. Jørgensen, Chem. Eur. J., 2010, 16, 28; e) S. Sobhani and Z. Tashrifi, Tetrahedron, 2010, 66, 1429; f) C. S. Demmer, N. Krogsgaard-Larsen and L. Bunch, Chem. Rev., 2011, 111, 7981.

3 For selected examples, see: a) D. V. Patel, K. Rielly-Gauvin and D. E. Ryono, Tetrahedron Lett., 1990, 31, 5587; b) D. V. Patel, K. Rielly-Gauvin and D. E. Ryono, Tetrahedron Lett., 1990, 31, 5591; c) B. Stowasser, K.-H. Budt, L. Jian-Qi, A. Peyman and D. Ruppert, 
Tetrahedron Lett., 1992, 33, 6625; d) J. A. Sikorski, M. J. Miller, D. S. Braccolino, D. G. Cleary, S. D. Corey, J. L. Font, K. J. Gruys, C. Y. Han, K. C. Lin, P. D. Pansegrau, J. E. Ream, D. Schnur, A. Shah and M. C. Walker, Phosphorus, Sulfur Silicon Relat. Elem., 1993, 76, 375; e) P. Page, C. Blonski and J. Périé, Bioorg. Med. Chem., 1999, 7, 1403; f) Y. Kobayashi, A. D. William and Y. Tokoro, J. Org Chem., 2001, 66, 7903; f) Y.-h. Koh, J. H. Shim, J. Z. Wu, W. Zhong, Z. Hong and J.-L. Girardet, J. Med. Chem., 2005, 48, 2867.

4 For selected examples, see: a) J. G. Allen, F. R. Atherton, M. J. Hall, C. H. Hassall, S. W. Holmes, R. W. Lambert, L. J. Nisbet and P. S. Ringrose, Antimicrob. Agents Chemother., 1979, 15, 684; b) F. R. Atherton, M. J. Hall, C. H. Hassall, R. W. Lambert, W. J. Lloyd and P. S. Ringrose, Antimicrob. Agents Chemother., 1979, 15, 696; c) D. V. Patel, K. Rielly-Gauvin, D. E. Ryono, C. A. Free, W. L. Rogers, S. A. Smith, J. M. DeForrest, R. S. Oehl and E. W. Petrillo Jr., J. Med. Chem., 1995, 38, 4557; d) Aminophosponic and aminophosphinic acids: Chemistry and Biological Activity, (V. P. Kukhar and H. R. Hudson (Eds.)), Wiley, Chichester, 2000; e) J. Huang and R. Chen, Heteroat. Chem., 2000, 11, 480.

5 For the pioneering enantioselective hydrophosphonylation of aldehyde using metal catalysts, see: a) T. Arai, M. Bougauchi, H. Sasai and M. Shibasaki, J. Org. Chem., 1996, 61, 2926; b) H. Sasai, M. Bougauchi, T. Arai and M. Shibasaki, Tetrahedron Lett., 1997, 38, 2717.

6 For additional remarkable examples of metal catalysedhydrophosphonylation reaction of aldehydes, see also: a) D. M Cermak, Y. Du and D. F. Weimer, J. Org. Chem., 1999, 64, 388; b) J. P. Duxbury, J. N. D. Warne, R. Mushtaq, C. Ward, M. Thornton-Pett, M. Jiang, R. Greatrex and T. P. Kee, Organometallics, 2000, 19, 4445; c) B. Saito and T. Katsuki, Angew. Chem. Int. Ed., 2005, 44, 4600; d) B. Saito, H. Egami and T. Katsuki, J. Am. Chem. Soc., 2007, 129, 1978; e) X. Zhou, X. Liu, X. Yang, D. Shang, J. Xin and X. Feng, Angew. Chem. Int. Ed., 2008, 47, 392; f) J. P. Abell and H. Yamamoto, J. Am. Chem. Soc., 2008, 130, 10521; g) F. Yang, D. Zhao, J. Lan, P. Xi, L. Yang, S. Xiang and J. You, Angew. Chem. Int. $E d$., 2008, 47, 5646; h) K. Suyama, Y. Sakai, K. Matsumoto, B. Saito and T. Katsuki, Angew. Chem. Int. Ed., 2010, 49, 797: i) P. Muthupandi and G. Sekar, Org. Biomol. Chem., 2012, 10, 5347.

7 For selected examples, see: a) D. L. Pompliano, E. Rands, M. D Schaber, S. D. Mosser, N. J. Anthony and J. B. Gibbs, Biochemistry, 1992, 31, 3800; b) M. Tao, R. Bihovsky, G. J. Wells and J. P. Mallamo, J. Med. Chem., 1998, 41, 3912; c) M. L. Peters, M. Leonard and A. A. Licata, Cleveland Clin. J. Med., 2001, 68, 945; d) R. Snoeck, A. Holý, C. Dewolf-Peeters, J. Van Den Oord, E. De Clercq and G. Andrei, Antimicrob. Agents Chemother., 2002, 46, 3356.

8 For selected examples, see: a) B. Iorga, F. Eymery and P. Savignac, Tetrahedron, 1999, 55, 2671; b) B. Kaboudin, Tetrahedron Lett., 2003, 44, 1051; c) H. Firouzabadi, N. Iranpoor and S. Sobhani, Synth. Commun., 2004, 34, 1463; d) H. Firouzabadi, N. Iranpoor, S. Sobhani and Z. Amoozgar, Synthesis, 2004, 1771; e) V. V. Nesterov and O. I. Kolodiazhnyi, Tetrahedron, 2007, 63, 6720.

9 For selected examples, see: a) J. Zhu and X. Lu, Tetrahedron Lett., 1987, 28, 1897; b) E. Öhler and S. Kotzinger, Synthesis, 1993, 497; c) J. F. Koszuk, Synth. Commun., 1995, 25, 2533; d) H. Shabany and C. D. Spilling, Tetrahedron Lett., 1998, 39, 1465 .
10 a) H. Wynberg and A. A. Smaardijk, Tetrahedron Lett., 1983, 24, 5899; b) A. A. Smaardijk, S. Noorda, F. V. Bolhuis and H. Wynberg, Tetrahedron Lett., 1985, 26, 493.

11 a) D. Uraguchi, T. Ito and T. Ooi, J. Am. Chem. Soc., 2009, 131, 3836. For organocatalytic enantioselective examples of addition using ketones, see also: b) F. Wang, X. Liu, X. Cui, Y. Xiong, X. Zhou and X. Feng, Chem. Eur. J., 2009, 15, 589; c) D. Uraguchi, T. Ito, S. Nakamura and T. Ooi, Chem. Sci., 2010, 1, 488.

12 D. Pettersen, M. Marcolini, L. Bernardi, F. Fini, R. P. Herrera, V. Sgarzani and A. Ricci, J. Org. Chem., 2006, 71, 6269.

13 For pioneering examples using imines in the organocatalytic Pudovik reaction, see also: a) G. D. Joly and E. N. Jacobsen, J. Am. Chem. Soc., 2004, 126, 4102; b) T. Akiyama, H. Morita, J. Itoh and K. Fuchibe, Org. Lett., 2005, 7, 2583.

14 R. P. Herrera, D. Roca-López and G. Navarro-Moros, Eur. J. Org. Chem., 2010, 1450

15 Hydrogen Bonding in Organic Synthesis. (P. M. Pihko (Ed.)), WileyVCH: Weinheim, 2009

16 a) G. Dessole, R. P. Herrera and A. Ricci, Synlett, 2004, 2374; b) R. P. Herrera, V. Sgarzani, L. Bernardi and A. Ricci, Agew. Chem. Int. Ed., 2005, 44, 6576; c) D. Pettersen, R. P. Herrera, L. Bernardi, F. Fini, V. Sgarzani, R. Fernández, J. M. Lassaletta and A. Ricci, Synlett, 2006, 239; d) L. Bernardi, F. Fini, R. P. Herrera, A. Ricci and V. Sgarzani, Tetrahedron, 2006, 62, 375; e) R. P. Herrera, D. Monge, E. Martín-Zamora, R. Fernández and J. M. Lassaletta, Org. Lett., 2007, 9, 3303; f) E. Marqués-López and R. P. Herrera, An. Quim., 2009, 105, 5-12; g) A. Alcaine, E. Marqués-López, P. Merino, T. Tejero and R. P. Herrera, Org. Biomol. Chem., 2011, 9, 2777; h) E. Marqués-López, A. Alcaine, T. Tejero and R. P. Herrera, Eur. J. Org. Chem., 2011, 3700; i) E. Marqués-López and R. P. Herrera, in New Strategies in Chemical Synthesis and Catalysis. (B. Pignataro (Ed.)), Wiley-VCH, 2012, pp 175-199.

17 See supporting information for additional results.

18 CCDC-953498 (4ee) and CCDC-953499 (4eg) contain the supplementary crystallographic data for this paper. These data can be obtained free of charge from the Cambridge Crystallographic Data Centre via ww.ccdc.cam.ac.uk/data request/cif.

19 For interesting reviews, see: a) J. Alemán, A. Parra, H. Jiang and K. A. Jørgensen, Chem. Eur. J., 2011, 17, 6890; b) R. I. Storer, C. Aciro and L. H. Jones, Chem. Soc. Rev., 2011, 40, 2330.

20 For reviews about bifunctional organocatalysts, see: a) H. Miyabe and Y. Takemoto, Bull. Chem. Soc. Jpn., 2008, 81, 785; b) S. J. Connon, Chem. Commun., 2008, 2499.

21 B. Springs and P. Haake, J. Org. Chem., 1977, 42, 472.

22 We were not able to achieve good results in terms of enantioselectivity in our model reaction neither using the same catalyst (Quinine) employed by Wynberg and co-workers, ${ }^{10}$ nor using Cinchonidine. $^{17}$

23 For a further transesterification extending the scope, see: a) J. Szewczyk, B. Lejczak and P. Kafarski, Synthesis, 1982, 409; b) J. Grembecka, A. Mucha, T. Cierpicki and P. Kafarski, J. Med. Chem., 2003, 46, 2641.

24 A. R. Katritzky, B. V. Rogovoy and A. Y. Mitrokhin, Arkivoc, 2002, (xiii), 17.

25 X. Zhu, S. Wang, S. Zhou, Y. Wei, L. Zhang, F. Wang, Z. Feng, L. Guo and X. Mu, Inorg. Chem., 2012, 51, 7134 